\title{
Energy Packet Networks: Smart Electricity Storage to Meet Surges in Demand
}

\author{
Erol Gelenbe FIEEE FACM \\ Member of Académie des Technologies (France) \\ Dept. of Electrical \& Electronic Engineering \\ Imperial College, London SW7 2BT, UK \\ e.gelenbe@imperial.ac.uk
}

\begin{abstract}
When renewable energy is used either as a primary source, or as a back-up source to meet excess demand, energy storage becomes very useful. Simple examples of energy storage units include electric car batteries and uninterruptible power supplies. More sophisticated examples include dams into which water may be pumped when renewable sources of electricity such as wind or photovoltaic are plentiful. Drawing an analogy between computer networks which store and forward and download data, and electrical networks that furnish energy both from direct production and storage, we suggest a model of Energy Packet Networks (EPNs) which store and forward quantised energy units to and from a large range of devices. In addition to being a model for networks of energy storage units, energy suppliers and consumers, an EPN can be an appropriate framework for energy harvesting networks at the micro-scale, and bio-nano scale energy networks. A mathematical model for EPNs is suggested and we analyse two examples using a probability model. We first consider the case where stored energy and a fast ramp-up source are used to meet random energy surges assuming an unlimited storage unit and a random flow of renewable energy, and compute the probability that all requests are satisfied, or that some cannot be satisfied, and the spillover rate of renewable energy. Then we analyse the effect of an imperfect communication and computer-control system where message losses and delays degrade the energy system performance.
\end{abstract}

\section{Keywords}

Energy Packet Networks, Smart Grid, Network Control of Energy Flow, On-Demand Energy Dispatching, Storing Renewable Energy, Store and Forward Energy

\section{INTRODUCTION}

Energy markets in have evolved from having a small number of suppliers to more flexible markets where consumers are able to select energy suppliers, while suppliers them-

Permission to make digital or hard copies of all or part of this work for personal or classroom use is granted without fee provided that copies are not made or distributed for profit or commercial advantage and that copies bear this notice and the full citation on the first page. To copy otherwise, to republish, to post on servers or to redistribute to lists, requires prior specific permission and/or a fee.

Simutools 2012, March 19-23, Desenzano del Garda, Italy

Copyright (c) 2012 ICST 978-1-936968-47-3

DOI 10.4108/icst.simutools.2012.247805 selves may be able to dynamically select the producers and the types of energy sources that they use. These trends are driven both by the push from public regulators for more competition in energy supply, by incentives to turn to by renewable energy sources (RES) which are becoming more plentiful, and by the introduction of smart meters and smart grid technologies which allow a far greater flexibility in matching supply and demand. The basic enabling technology in all of this are data networks and distributed computer based decision systems.

The use of RES such as solar panels, wind power, tidal flows, hydroelectric sources and geothermal power all introduce new dynamic time variations which typically unpredictable and sometimes aperiodic. While RES introduce an unpredictable element at the supply side, time varying, but more predictable characteristics are present at the demand side of the energy market. Ideally one should be able to tune the demand side $[23,15]$ to meet the supply. Forecasting demand [5] using detailed publicly available data [25] has received considerable attention. However, to smooth out this interaction between dynamic supply and demand, energy storage systems such as the batteries of electric vehicles, various forms of electronic uninterruptible power supplies (UPS), compressed gas depots (GD), dams and water towers (WT), and electric cars, can offer new opportunities to provide multiple energy buffers between fluctuating energy supply and demand. Thus in the face of unpredictable and highly variable renewables such as wind $[18,26]$, storage technologies are an appealing means of smoothing the supply-demand equation and have thus received considerable attention [14, 32, 34, 37, 41].

Pursuing the idea of storing and forwarding energy on demand, we propose to exploit an interesting analogy between an energy request and distribution system and data networks. Indeed:

- One can think of a consumer's request for energy as a "demand for video" or for a download from a web server, where the content emulates the energy and the flow of data across the computer network emulates the flow of electrical current from the source(s) to the $\operatorname{sink}(\mathrm{s})$.

- Intermediate store-and-forward buffers where packets are stored can be viewed as centres where energy is stored, such as batteries or UPSs, or other energy storage devices such as dams or WTs with pumps and 
water turbine generators or GDs with pumps and gas turbines for energy generation.

Thus this paper thus follows upon our recent proposal of the concept of an Energy Packet Network (EPN) [35] which is a virtualised dynamic fine-grained energy storage and distribution system based on "energy packets" (EP). An EPN includes:

- A physical energy system (PES) that combines intermittent renewable energy sources, other sources that can be turned on or ramped up or ramped down on demand, and energy storage units,

- A variable set of intermittent industrial, business or consumer requests for energy.

- The scheduling of energy flows, that resembles a data network with flows of "energy packets" operating in "energy store and forward mode (ESF)" or in "energy asynchronous transfer mode (EATM)",

- A virtualised representation of the physical system (VES) using networking hardware and software that emulates the flow of energy, and senses these flows directly at the key components (sources, sinks, energy transport network, energy storage units) of the PES,

- A computer based energy dispatching system (EDS) that selects the energy flows to be dispatched to the requests using the observations of the PES and smart emulation in the VES.

The aim of an EPN is to provide a flexible, economical and efficient response to dynamic energy needs in the presence of time-varying energy sources. The EPN can store energy originating from from RES when it is cheap or plentiful, and complement traditional sources of energy during demand peaks. EPNs can serve both as a way to make RES more practical, and to reduce the cost of energy for consumers and to the suppliers, reduce the peak load of high and low voltage lines and reduce the need for over-dimensioned electric transport lines and transformers. An EPN can serve different purposes:

- It can be used to provide real-time information about the requests on the one hand, and the sources of energy on the other, so that an electrical energy network may be managed in real-time to provide lower overall costs, lower overall CO2 imprint, and greater reliability and security, and/or

- It may be used to schedule the flow of current to and from electricity storage units, based on availability and demand,

- It may also be useful for real-time scheduling of energy demand so as to meet certain desirable objectives, e.g. scheduling electric heating in a large building in a round-robin or random manner among different flats or rooms, or among different rooms on the same floor, so that peaks and troughs in energy consumption are avoided and a constant or slowly time varying overal power flow is maintained, and for scheduling the usage of appliances within time periods when energy costs are lower.

EPNs can also map down to the bio-nano and micro-level:

- One can consider living cells as consumers of energy in the form of (say) sugar molecules, while other cells generate the sugar molecules, others store them, and the capillary system conveys the molecules (energy packets) from storage and generator cells to the consumers, such as the neurons which carry out brain computations. This representation would then allow the modeling of neurons, glial cells and the capillaries which attract blood flow with the needed energy to the neurons that are carrying out computations.

- At the micro-level one can consider MEMS or photovoltaic energy harvesters, with micro-batteries and capacitors where energy is stored, and the electrons themselves and other charged particles are the energy packets that flow through the network of harvesters, storage units and micro/nano-processing devices.

\subsection{Architecture of an Energy Packet Network}

We see that an Energy Packet Network is an integrated adaptive electrical energy storage, distribution and consumption system. In addition to a conventional scheme for distributing energy based on instantaneous flow of current towards points of energy consumption, it offers smart management of requests and dispatching of EPs to meet the demands of individual or commercial consumers and various autonomous electrical devices and appliances, based on smart dynamic generation, storage and dispatching of electric power. Such systems may be particularly well adapted to environments where RES are common, and where effective means for storing energy, such as electric cars and UPS, are available.

Such a system may include many geographically distributed renewable energy sources, as well as conventional sources of energy that originate from fossil and nuclear power plants, and a distribution network, together with distributed energy storage facilities and distributed energy consumption sinks. We can imagine that many of the consumption sinks would be coupled at close distance with one or more storage facilities.

The flow of energy in the EPN is controlled by Smart Energy Dispatching Centres (SEDCs) which receive requests from both the consumers and storage centres, and optimise the energy flows by making the best use of renewable energy sources and existing pricing policies, while satisfying consumer demands and minimising peak energy flows through buffering and scheduling of energy. SEDCs are computer control centres which receive information and make dispatching decisions from/to to all other system components via data communication networks.

The basic unit of energy in this system, an EP, can be viewed as a pulse of power that lasts a certain time; it constitutes the basic energy delivery unit of our system, say in KWH. We denote the energy content of such a packet by $E \mathrm{KWH}$, 
and this quantity must be small enough to be close to the smaller energy needs of consumers, e.g. lighting a house for a few hours, or operating the coffee pot or microwave oven during the morning breakfast, yet large enough to be measurable and billable as a significant and useful quantity of energy.

The discretised and dynamically buffered nature of this system which integrates Quantised Requests, Generation, Storage and Distribution of Electric Power, motivates our choice of its name: the Energy Packet Network.

\section{ENERGY PACKET NETWORK MODEL}

Although the system we consider is intuitively appealing, its value can only reside in its potential as a better means of delivering electric power. Thus we need to examine how such a system may be modelled and how its performance advantages may be shown through system analysis. Thus in this section we will develop a model representation for EPNs. The theoretical framework that we will use is a class of queueing networks [1] known as G-networks [4, 6, 9], which have the advantage of (a) incorporating the flow of the discretised commodity of interest (the EPs) and its storage in STs (the queues), (b) representing the choices that are made regarding the distribution of these flows through the EPN (via routing probabilities), (c) and most importantly also representing the flow of data that is needed for control decisions (for instance from the consumer demands to the storage and sources of energy supplies upstream) and the direct coupling of these data flows of requests with the decisions to transfer EPs towards the consumers. Thus in this model the EPs constitute the "ordinary customers" of the queueing network, the STs are the queues, the external arrivals of EPs are the energy produced by different sources of energy, and the G-network's "triggers" are the data flows regarding requests made by consumers or by STs that request that their energy buffers be replenished.

The system we consider has $N$ energy sources (ES) $s(i)$, each with a stochastic energy generation rate $G(i)$ in $\mathrm{EP} / \mathrm{sec}$, for $i \in\{1, \ldots, N\}$, where $G(i) \leq G_{M}(i)$ with $G_{N}(i)$ being the maximum energy production rate of ES $i$. With each source $i$ we will associate a generating state $g(i, t)$ at time $t \geq 0$, which varies between 0 and $G_{M}$ so that the source may have different levels of energy generation. The energy sources are either renewable, in which case $s(i) \in R$, or they are conventional in which case $s(i) \in C$.

The system has M energy storage centres (ST) $S(j)$ each with finite storage capacity $B(j)$ in EPs, $j \in\{1, \ldots, M\}$. Each storage centre has an energy conversion efficiency $(1-$ $\left.L_{j}\right), 0 \leq L_{j}<1$ at its input so that on average the arrival of $B^{\prime}(j)$ energy packets to ST $j$ results in the storage of $B(j)=L_{j} \cdot B^{\prime}(j)$ EPs. It also has an energy loss rate which is $\mu_{j}$ per unit time so that if storage is not replenished, a storage centre initially containing $B(j)$ EPs will be depleted of energy on average after a time $B(j) / \mu_{j}$.

We also have $K$ energy consumption centres (CC); each one may contain a local energy storage facility with an instantaneous storage value of $b(k)$. Some CCs may also have no storage capability. A consumption centre has a stochastic consumption rate of $m_{k}$ in EPs/sec and we assume that when consumption is taking place, the energy that is buffered at the consumption centre is being depleted. Note that a modern Data Centre with its uninterruptible power supply (UPS) is a realistic example of a CC.

The $N$ sources, $M$ storage centres and the $K$ consumption centres are interconnected by an energy distribution network (EDN) represented by a graph, so that link $(u, v)$ of the directed graph represents a power line that has an energy transport capacity $C(u, v)$ which is the maximum amount of power that can be transferred instantaneously from node $u$ to node $v$. Thus a link in the energy transportation network will be represented by an unbounded capacity input buffer, followed by a rate of exit that cannot exceed $C(u, v)$ in $\mathrm{EP} / \mathrm{sec}$. In addition the link will have an efficiency $0<(1-l(u, v)) \leq 1$ which is the fraction of energy introduced into the link that actually reaches the destination.

The nodes of the EDN may be production nodes, consumption or storage nodes, or transduction nodes which can have many inputs and outputs. A transduction node $u$ does not generate or store energy but dispatches it from one or more nodes to one or more other nodes; it has a transduction power capacity $T(u)$ so that for any successor node $v$ we have $\sum_{v} C(u, v) \geq T(u)$ and for any predecessor $v$ we have $\sum_{v} C(v, u) \leq T(u)$. Thus the incoming link capacities to a transduction node cannot exceed its own capacity, while the transduction node's outgoing links need to have a total capacity that exceeds its own capacity. The transduction node $u$ will also have an efficiency $0<(1-l(u)) \leq 1$ so that a fraction $l(u)$ of the power that it receives is wasted.

Each CC will send its energy requests to a Smart Dispatching Centre (SDC). SDCs are computational facilities that are interconnected to other system components via a computercommunications network. Each SDC keeps track of the energy needs and requests in an area and assigns flows from the STs and ESs to the CCs. The SDCs also send requests to the ESs so that they may replenish the STs.

The SDCs' role is to satisfy the requests of the CCs and to make sure that STs have a standby capacity to meet unexpected needs. The SDCs will typically use pricing policies help the STs replenish their power at the best price, and also when energy from photovoltaic, wind or from other renewable sources is more readily available. The SDCs also attempt to maintain a flow of EPs across the EDN which is as low as possible so that energy traffic peaks are avoided and the EDN avoids saturation. Indeed one of the overarching objectives of the energy packet system is to be able to operate reliably with the lowest overall load being carried by the EDN.

\section{ENERGY PACKET NETWORK PERFOR- MANCE}

The performance model we propose focuses on the energy flows in the system and allows us to evaluate many of the quantities of interest including the amount of energy being stored in each part of the EPN, the average flow of power on each of the links of the transport network, the probability that a request for power is not satisfied, and the average delay between the time that a request is made until the in- 
stant at which the power delivery begins. The model can also be used to estimate the performance risks and enhancements offered by an EPN in terms of performance measures of interest. Since it incorporates both the power transport network and the EPNs communication network, it can also be used to focus on system failures which may occur because of failures in the communication network.

As simple examples of such performance models we will consider two different system models:

- The first system considers a steady source of energy (e.g. a thermal or nuclear power plant), a source of renewable energy, a storage system (e.g. a dam), and a fast ramping source of energy that is called upon when the energy needs exceed the available energy from the steady source, the renewal energy and the storage system; here we assume that ICT based communications to communicate the needs of the system between the different units are carried out "perfectly" without loss of information and without delay.

- The second performance model investigates the effect of communication message losses on a system which uses energy storage from renewable energy as a backup source of energy.

\subsection{Unlimited Stored Energy Back-Up}

We consider a steady source of energy set at $c_{T}$ units of energy per unit time which has been selected as a result of energy demand forecasts and of the different system components, such as the storage unit, the renewable energy supply, back-up fast ramp energy devices which are available when all else fails.

The energy demand in excess of this value $c_{T}$ is represented by a sequence of units (e.g. Joules) which are spaced at random time intervals that are measured in microseconds or nano-seconds, resulting in a measurement rate of $D$ samples per second: we are discretising the measured power in "bins" of 1 units and each time that the demand exceeds the set supply value $c_{T}$ by 1 joules, then we register this request so that it may be met by the renewable source or the storage system. Thus the measured average power demand in excess of $c_{T}$ is $D$, for instance in Megawatts if $D$ is of the order of a "one sample per microsecond", but the instants when these excess requests occur are random. Thus these excess requests are being represented as "arrivals" in a random process.

We have a source of renewable energy (e.g. wind or phovoltaic) which generates a random supply of energy units at a rate $\lambda_{r}$. This energy is directed towards a storage unit (e.g. a dam, compressed air storage unit, water tower, battery) which can store at most $S$ energy units (e.g. Giga-Joules), and the stored energy is returned to meet demand when the demand exceeds the generation level set at $c_{T}$. The storage unit has a loss rate of $\mu$, e.g. through leakage from the storage dam, or leakage from the battery. The process of storing of the renewable energy also results in the loss of a fraction of $L$ of the energy during the conversion (e.g. from electricity generated by a renewable source to water that has been pumped into a dam), while the extraction and re-use of the energy from storage also results in a loss of a fraction $l$. Furthermore, when we are extracting the energy from storage, the rate at which it is extracted cannot exceed $\alpha$ in watts or megawatts. The maximum value $\alpha$ results from the fact that the storage unit, say a water dam, has a generator whose maximum energy production rate is $\alpha$ watts. Similarly, if the storage unit is a battery, it has a maximum output current and a fixed voltage, which together will determine the battery's maximum output power. Finally, if the storage unit is empty or unable to produce enough electricity to meet the demand then a fast ramp source of energy that produces at a rate $\beta$ is turned on.

A simple mathematical model for this system will include:

- A queue $Q R$ with a Poisson arrival process of rate $(1-L) \lambda_{r}$ and maximum capacity $S$, so that when the storage is full the incoming flow of renewable energy is lost, which is also known as "spillover",

- A second queue $Q D$ with Poisson arrival process of rate $D$ representing the pent-up or unsatisfied instantaneous demand; when this queue is non-empty then it generates requests for "negative customers" [4] from $Q S$ which remove or destroy the unsatisfied demand from $Q D$. Furthermore, when there is not enough available renewable energy then a fast ramp

Using G-network theory in an approximate manner, and assuming that $S=\infty$, i.e. that the $Q R$ has unlimited capacity, we have the two following coupled equations for the stead-state probabilities $Q$ and $r$ that $Q D$, respectively $Q S$, contains at least one unit of energy demand or stored:

$$
Q=\frac{D}{r \alpha(1-l)+(1-r) \beta}
$$

and from the parameter

$$
q=\frac{\lambda_{r}(1-L)}{Q \alpha+\mu}
$$

we compute the probability that the storage unit is nonempty:

$$
r=q \frac{1-q^{S}}{1-q^{S+1}}
$$

When the energy storage capacity is finite $S<+\infty$, we can also estimate the spillover rate $\Lambda_{s}$ of renewable energy when the storage is full, which is:

$$
\Lambda_{s}=\lambda_{r} q^{S} \frac{1-q}{1-q^{(S+1)}}
$$

In this case, the average amount of stored energy is given by:

$$
<E_{S}>=\frac{q}{1-q}-\frac{(S+1) q^{S}}{1-q^{(S+1)}}
$$

When the energy storage unit has no leakage, i.e. $\mu=0$ equations (1) and (]ref2) will yield

$$
q=\frac{\rho_{r}}{\frac{D}{\beta}+\rho_{r}\left[1-(1-l) \frac{\alpha}{\beta}\right]}
$$


Define the load factor for the renewable energy storage centre to be $\rho_{r}=\lambda_{r}(1-L) / \alpha$. In the ideal case where the energy storage centre has unlimited capacity $S=+\infty$ and no loss during storage $\mu=0$ we obtain:

$$
\begin{aligned}
& Q=\min \left[1, \frac{D}{\beta}+\rho_{r}\left[1-(1-l) \frac{\alpha}{\beta}\right]\right] \\
& q=\min \left[1, \frac{\rho_{r}}{\frac{D}{\beta}+\rho_{r}\left[1-(1-l) \frac{\alpha}{\beta}\right]}\right]
\end{aligned}
$$

where $0 \leq Q \leq 1,0 \leq q \leq 1$. The objectives of the system as a whole are to satisfy the following properties:

- $Q$ should be as close to zero as possible so that the excess demand is always satisfied,

- $q$ should be as close to one as possible so that if there is excess demand then there is enough stored energy and sufficient conversion speed and efficiency so that the excess demand is rapidly satisfied.

If the energy storage capacity is unlimited and has no leakage, the average amount of stored energy is also easily computed from $q$ :

$$
<E>=\frac{\lambda_{r}(1-L) \frac{\beta}{\alpha}}{D-\lambda_{r}(1-L)(1-l)}
$$

provided that $\lambda_{r}(1-L)(1-l)<D$ which means that the net renewable energy rate is just under the excess demand. This of course means that for a total energy demand rate $d$, the continuous production rate $c_{T}$ is set parsimoniously so that it is below the level that would be needed $c_{T}<$ $d-\lambda_{r}(1-L)(1-l)$ to satisfy all of the demand and the system then is operating under the capacity required by the consumers.

\subsection{Imperfect Communication and Computer Control System}

Now suppose that when the demand exceeds the set generation rate $c_{T}$, a message is sent through a communication network to the energy storage centre and that the message may be lost with probability $(1-p)$. Furthermore when the message does arrive, the total delay between the instant when the message is sent and the power converter at the storage centre is an exponentially distributed random variable with rate $\delta$. Similarly, assume that if the energy storage centre is empty (of the energy source) then the consumers are aware of this and they turn to the fast ramp-up generator by sending a message through the communication network with the same probability of message loss and total delay for the generator to start producing the required energy.

We then have:

$$
Q^{\prime}=\frac{D}{p r^{\prime}\left(\alpha^{\prime}(1-l)+\left(1-r^{\prime}\right) \beta^{\prime}\right.}
$$

and from the parameter

$$
q^{\prime}=\frac{\lambda_{r}(1-L)}{Q^{\prime} p \alpha^{\prime}+\mu}
$$

where:

$$
\frac{1}{\alpha^{\prime}}=\frac{1}{\alpha}+\frac{1}{\delta}
$$

$$
\frac{1}{\beta^{\prime}}=\frac{1}{\beta}+\frac{1}{\delta}
$$

so that assuming we have unlimited energy storage capacity, we can easily estimate the effect of communication message loss and of computer control system delay on the probability that all energy needs are not satisfied by examining the ratio of $Q^{\prime}$ to $Q$ :

$$
\frac{Q^{\prime}}{Q}=\frac{1}{p} \frac{\frac{D}{\beta}\left(1+\frac{\beta}{\delta}\right)+\rho_{r}\left(1+\frac{\alpha}{\delta}\right)\left[1-(1-l) \frac{1+\frac{\delta}{\beta}}{1+\frac{\delta}{\alpha}}\right]}{\frac{D}{\beta}+\rho_{r}\left[1-(1-l) \frac{\alpha}{\beta}\right]}
$$

From this analysis we see that:

1. As the probability of reliable message delivery $p$ becomes smaller, then the probability $Q^{\prime}$ that the energy needs are not satisfied will grow.

2. Similarly if $\alpha<\beta$ which means that the source of renewable energy ramps up more slowly than the "fast" but undesirable energy source, then the probability that all energy needs are not satisfied is also sure to grow as $\delta$ becomes smaller, i.e.when the computer system responds more slowly.

\section{FUTURE WORK}

Starting from ideas we developed in a recent paper [35] regarding the analogy between store-and-forward packet networks that are the basis of the Internet, and geographically distributed energy networks that use storage units to respond to the troughs and peaks in demand and in renewable energy, this paper has investigated the performance characteristics of Energy Packet Networks using mathematical tools from queueing theory and G-networks. We have also investigated the impact of computer system and network performance and quality of service, and we have observed that ICT imperfections will directly impact the effectiveness of a networked energy storage and management system.

The use of Information and Communication Technologies for fast adaptive management of energy systems introduces additional liabilities and risks, including network security issues in the presence of malicious attacks $[12,21]$. These aspects need to be addressed in greater detail in future work. We also need to study carefully the impact on the energy system of standard performance and quality of service characteristics of the networks and computer systems that were introduced in Section 3.2.

In future research we also plan to investigate the use of smart routing techniques that have been developed recently to convey network traffic in the best possible way to meet the endusers' needs $[10,19]$, as well as to reduce energy wastage while data is being routed [29, 28]. Such techniques may prove to useful for conveying energy in this new area of adaptively optimised energy storage and management. Linking this approach to modern micro and nano energy harvesting networks will also be an interesting challenge.

\section{Acknowledgements}

The author gratefully acknowledges the support for this research from the Fit4Green European Union FP7 Project cofunded under ICT Theme: FP7-ICT-2009-4. 


\section{REFERENCES}

[1] E. Gelenbe and R. R. Muntz "Probabilistic Models of Computer Systems - Part I (Exact Results)", Acta Informatica, 7: 35-60, 1976.

[2] E. Gelenbe "Diffusion approximations, waiting times and batch arrivals", Acta Informatica, 12: 285-303, 1979.

[3] D. W. Bunn, E. Farmer (Eds.) "Comparative Models for Electric Load Forecasting", John Wiley \& Sons, 1985.

[4] E. Gelenbe and A. Stafylopatis "Global behaviour of homogeneous random neural systems", Applied Mathematical Modelling, 15 (10): 534-541, 1991.

[5] R. Ramanathan, R. Engle, C.W. Granger, F. Vahid-Araghi and C. Brace "Short-run forecasts of electricity loads and peaks", International Journal of Forecasting, 13 (2): 161-174, 1997.

[6] E. Gelenbe, A. Labed "G-networks with multiple classes of signals and positive customers", European Journal of Operations Research, 108 (2): 293-305, 1998.

[7] G. Winkler, C. Meisenbach, M. Hable, and P. Meier, "Intelligent energy management of electrical power systems with distributed feeding on the basis of forecasts of demand and generation", In CIRED 2001.

[8] D. J. Zack "Overview of wind energy generation forecasting", Tech. Rep., TrueWind Solutions, LLC, 2003.

[9] E. Gelenbe and J.-M. Fourneau "G-Networks with resets", Performance Evaluation, 49: 179-192, 2002.

[10] E. Gelenbe "Cognitive Packet Network" U.S. Patent No. 6804201 B1, Oct. 12, 2004.

[11] J. W. Taylor "Density forecasting for the efficient balancing of the generation and consumption of electricity", International Journal of Forecasting, 22 (4): 707-724, 2006.

[12] E. Gelenbe and George Loukas "A self-aware approach to denial of service defence", Computer Networks, 51 (5): 1299-1314, April 2007.

[13] J.R. Cancelo, A. Espasa, and R. Graffe "Forecasting the electricity load from one day to one week ahead for the spanish system operator", International Journal of Forecasting 24 (2): 588-602.

[14] M. Black and G. Strbac "Value of bulk energy storage for managing wind power fluctuations", IEEE Transactions on Energy Conversion, 22(1): 197-205, Mar. 2007.

[15] D. Infield, J. Short, C. Home, and L. Freris "Potential for domestic dynamic demand-side management in the UK", IEEE Power Engineering Society General Meeting, pp. 1-6, June 2007.

[16] V. Dordonnat, S. Koopman, M. Ooms, A. Dessertaine and J. Collet "An hourly periodic state space model for modelling French national electricity load", International Journal of Forecasting, 24 (4): 566-587, 2008.

[17] I. Sanchez "Adaptive combination of forecasts with application to wind energy", International Journal of Forecasting 24 (4): 679-693, 2008.

[18] J. Manwell, J. McGowan, and A. Rogers "Wind Energy Explained: Theory, Design and Application",
Wiley, 2009

[19] E. Gelenbe "Steps toward self-aware networks", Comm. ACM, Vol. 52 (7), pp. 66-75, 2009.

[20] A. Berl, E. Gelenbe, M. di Girolamo, G. Giuliani, H. de Meer, M.-Q. Dang, and K. Pentikousis "Energy-efficient Cloud Computing", The Computer Journal, Vol. 53 (7), pp. 1045-1051, 2010, doi:10.1093/comjnl/bxp080.

[21] G. Sakellari and E. Gelenbe, "Demonstrating cognitive packet network resilience to worm attacks", Proc. ACM Conference on Computer and Communications Security, pp. 636-638, 2010.

[22] The MeRegio Project. http://www.meregio.de/en/. 2011. Center for Renewable Energy Sources. http://www.cres.gr/.

[23] H. Berthold, M. Boehm, L. Dannecker, F.-J. Rumph, T.B. Pedersen, C. Nychtis, H. Frey, Z. Marinsek, B. Filipic and S. Tselepis "Exploiting renewables by request-based balancing of energy demand and supply", Proc. 11th IAEE European Conference, 2010.

[24] MIRACLE Project 2010. MIRACLE Project Website. MIRACLE Project. http://www. miracle- project.eu.

[25] Nationalgrid UK 2010. Metered half-hourly electricity demands. Nationalgrid UK. http://www. nationalgrid.com/uk/Electricity/Data/Demand+Data/.

[26] NREL 2010. Wind Integration Datasets. NREL. http://www.nrel.gov/wind/ integrationdatasets/.

[27] E. Gelenbe and C. Morfopoulou "Routing and G-Networks to optimise energy and Quality of Service in packet networks", Energy-Efficient Computing and Networking, Lecture Notes of the Institute for Computer Sciences, Social Informatics and Telecommunications Engineering, 54, Part 4, 163-173, Springer, 2011, DOI : $10.1007 / 978-3-642-19322-4{ }_{1} 8$.

[28] E. Gelenbe and T. Mahmoodi, "Energy-Aware Routing Protocol in the Cognitive Packet Network", International Conference on Smart Grids, Green Communications, and IT Energy-aware Technologies (Energy 2011), Venice, Italy, 22-27 May 2011, ISBN : $978-1-61208-006-2$.

[29] E. Gelenbe and C. Morfopoulou "A framework for energy aware routing in packet networks", The Computer Journal 54 (6): 850-859, 2011.

[30] Dinorwig power station. First Hydro Company, URL: http://www.fhc.co.uk/dinorwig.htm.

[31] E. Bitar, R. Rajagopal, P. Khargonekar, and K. Poolla "The role of co-located storage for wind power producers in conventional electricity markets", Proc. American Control Conference (ACC), 3886-3891, July 2011.

[32] K. Chandy, S. Low, U. Topcu, and H. Xu. A simple optimal power flow model with energy storage. In 49th IEEE Conference on Decision and Control (CDC), 1051-1057, Dec. 2010.

[33] D. Gayme and U. Topcu "Optimal power flow with distributed energy storage dynamics", Prpc. American Control Conference, 2011.

[34] P. Grünewald, T. Cockerill, M. Contestabile, and P. Pearson "The role of large scale storage in a GB low carbon energy future: Issues and policy challenges", 
Energy Policy, 39(9): 4807-4815, 2011.

[35] E. Gelenbe "Energy Packet Networks", Keynote Talk, GreenNets 2011 : The First ICST International

Conference on Green Communications and Networking, Colmar, France, Oct. 5-7, 2011.

[36] H. Oh "Optimal planning to Include Storage Devices in Power Systems", IEEE Transactions on Power Systems. 26 (3): 1118-1128, Aug. 2011.

[37] G. Sinden "Characteristics of the UK wind resource: long-term patterns and relationship to electricity demand", Energy Policy, 35 (1):112-127, 2007.

[38] H.-I. Su and A. E. Gamal "Modeling and analysis of the role of fast-response energy storage in the smart grid", Proceedings of the Forty-Ninth Annual Allerton Conference on Communication, Control, and Computing, University of Illinois at Urbana-Champaign, Sept. 2011.

[39] H.-I. Su and A. E. Gamal "Modeling and analysis of the role of fast-response energy storage in the smart grid", CoRR, abs/1109.3841, 2011.

[40] N. Wade, P. Taylor, P. Lang, and P. Jones "Evaluating the benefits of an electrical energy storage system in a future smart grid", Energy Policy, 38 (11): 7180-7188, 2010. 